\title{
Multiple subcutaneous inflammation, osteolysis, and polyarthritis
}

\author{
Yasuhiko Takechi • Tetsuya Shinozaki • \\ Toshio Fukuda • Kazuyoshi Asami • \\ Takashi Yanagawa $\cdot$ Kenji Takagishi
}

Published online: 23 February 2010

(C) ISS 2010

\section{Diagnosis}

Subcutaneous and intraosseous fat necrosis associated with pancreatic acinar cell carcinoma

\section{Discussion}

Chronic and acute pancreatitis, adenocarcinoma, and acinar cell carcinoma of the pancreas can cause a syndrome characterized by fever, polyarthritis, subcutaneous and intraosseous fat necrosis, and increased serum lipase activity [1-5]. However, diagnosis is difficult when the patient has no abdominal symptoms. Histology of the subcutaneous fat tissue and the lytic bone show only nonspecific inflammation and necrosis, which are insufficient to establish a diagnosis. Skin lesions, as a manifestation of subcutaneous fat necrosis from pancreatic disease,

The case presentation can be found at doi:10.1007/s00256-010-0876-6.

Y. Takechi $\cdot$ T. Shinozaki $(\bowtie) \cdot$ T. Yanagawa $\cdot$ K. Takagishi Department of Orthopaedic Surgery,

Gunma University Graduate School of Medicine, 3-39-22 Showa,

Maebashi, Gunma 371-8511, Japan

e-mail: tshinoza@showa.gunma-u.ac.jp

T. Fukuda

Department of Laboratory Sciences \& Pathology, Gunma University School of Health Sciences,

Maebashi, Japan

\section{K. Asami}

Department of Orthopaedic Surgery,

Maebashi Red Cross Hospital,

Maebashi, Japan can be distributed widely throughout the body. However, these findings are insufficient to support a definite diagnosis, especially at their onset. Eosinophilia is common in patients with pancreatic disease and subcutaneous fat necrosis [2]. However, laboratory data alone are insufficient for a definite diagnosis without abdominal symptoms. Whole-body computed tomography (CT) scanning was performed to clarify the etiology of the fever in this patient and revealed pancreatic acinar cell carcinoma and liver metastases. Acinar cell carcinoma of the pancreas often occurs in middle-aged men with nonspecific symptoms. Arthritis and subcutaneous fat necrosis were observed in $16 \%$ of those patients. Half of the patients had metastases at presentation; an additional 23\% subsequently developed metastases. One-year and 3-year survival rates were, respectively, $57 \%$ and $26 \%$ [6]. Radical therapy for the patient, even after definite diagnosis when extra-abdominal symptoms appear at presentation and after a long lapse in making the diagnosis, is almost impossible to prevent a rapid and fatal outcome of this disease. Indeed, an autopsy revealed pancreatic carcinoma in a patient with polyarthritis and subcutaneous fat necrosis [7]. Abdominal CT would show the tumor in the pancreas whether abdominal symptoms exist or not. However, it would be unusual to perform abdominal $\mathrm{CT}$ of patients with fat necrosis or polyarthritis unless we keep this condition in mind $[3,6]$. High serum lipase levels also facilitate a definite diagnosis $[3,4,6]$. So that earlier diagnoses can be made following appropriate therapy [4], we must bear in mind the possibility of subcutaneous and intraosseous fat necrosis attributable to pancreatic disorders, including acinar cell carcinoma.

Fat necrosis associated with pancreatitis or pancreatic carcinoma was inferred as resulting from the liberation of lipase and other enzymes into the circulation directly or via lymphatic channels [8, 9]. Many case reports have 
described the elevation of pancreatic serum enzymes such as amylase [8, 9], trypsin, and, especially, lipase [1, 3-5]. A possible cause of arthritis in patients with pancreatic disease is free fatty acids, which might cause arthritis when released into the joints [1]. Simkin et al. [10] explained the elevation of free fatty acids in the synovial fluid of the inflammatory joint in those patients. However, some patients with pancreatitis and subcutaneous fat necrosis show accompanying pleural and pericardial effusions aside from arthritis [8]. Immunofluorescence studies of the inflammatory pleura showed that the staining of immunoglobulins $\mathrm{G}$ and $\mathrm{C} 3, \mathrm{C} 1 \mathrm{q}, \mathrm{C} 3, \mathrm{C} 4, \mathrm{C} 5$, and CH50 was also reduced in serum and in pleural and pericardial effusions. Based on these facts, immune complex formation, except for the free serum lipase, might be the pathogenesis of inflammation associated with pancreatic disease [8].

\section{References}

1. Ferrari R, Wendelboe M, Ford PM, Corbett WEN, Anastassiades TP. Pancreatic arthritis with periarticular fat necrosis. J Rheumatol. 1993;20:1436-7.
2. Mullin GT, Caperson Jr EM, Crespin SR, Williams Jr RC. Arthritis and skin lesions resembling erythema nodosum in pancreatic disease. Ann Intern Med. 1968;68:75-87.

3. Radin DR, Colletti PM, Forrester DM, Tang WW. Pancreatic acinar cell carcinoma with subcutaneous and intraosseous fat necrosis. Radiology. 1986;158:68-7.

4. van Klaveren RJ, de Mulder PH, Boerbooms AM, van de Kaa CA, van Haelst UJ, Wagener DJ, et al. Pancreatic carcinoma with polyarthritis, fat necrosis, and high serum lipase and trypsin activity. Gut. 1990;31:953-5.

5. Rodriguez M, Lopez GL, Prieto P, Fernandez L, Willisch A, Arce M. Massive subcutaneous and intraosseous fat necrosis associated with pancreatitis. Natural evolution of the radiographic picture. Clin Rheumatol. 1997;16:199-203.

6. Klimstra DS, Heffess CS, Oertel JE, Rosal J. Acinar cell carcinoma of the pancreas. A clinicopathologic study of 28 cases. Am J Surg Pathol. 1992;16:815-37.

7. Virshup AM, Sliwinski AJ. Polyarthritis and subcutaneous nodules associated with carcinoma of the pancreas. Arthritis Rheum. 1973;16:388-92.

8. Potts DE, Mass MF, Iseman MD. Syndrome and pancreatic disease, subcutaneous fat necrosis and polyserositis. Case report and review of literature. Am J Med. 1975;58:417-23.

9. Phillips Jr RM, Sulser RE, Songcharoen S. Inflammatory arthritis and subcutaneous fat necrosis associated with acute and chronic pancreatitis. Arthritis Rheum. 1980;23:355-3602.

10. Simkin PA, Brunzell JD, Wisner D, Flechtner JJ, Cardin JS, Willkens RF. Free fatty acid in the pancreatic arthritis syndrome. Arthritis Rheum. 1983;26:127-32. 